\title{
STUDY OF POLYSOMNOGRAPHY IN CURE POST-TUBERCULOSIS PATIENTS
}

\author{
Bhise Dhondiba Haribhau ${ }^{1}$
}

\section{HOW TO CITE THIS ARTICLE:}

Bhise Dhondiba Haribhau. "Study of Polysomnography in Cure Post-Tuberculosis Patients". Journal of Evolution of Medical and Dental Sciences 2015; Vol. 4, Issue 67, August 20; Page: 11616-11625,

DOI: $10.14260 /$ jemds/2015/1676

ABSTRACT: Patients with obstructive sleep apnea (OSA) have various Comorbidities like cardiovascular, cerebrovascular diseases and metabolic disorders. There is an association between pulmonary hypertension and obstructive sleep apnea. Obstructive sleep apnea is associated with nocturnal arterial oxygen desaturation. During apnea the increased vagal tone induces hypoxia and by mechanical effects of obstructive sleep apnea associated with intrathoracic pressure swings leads to bradycardia and acute increase in pulmonary artery pressure. Patients with coronary artery disease may develop myocardial ischemia during apneas. Post-tuberculosis patients can lead to pulmonary sequelae that are characterized by impairments in the bronchial and parenchymal structure. The structural changes include bronchovascular distortions, bronchiectasis, emphysema and fibrosis. This leads to greater ventilation perfusion (V/Q) mismatch and hypoxia.

KEYWORDS: Post-Tuberculosis, Post-infective pulmonary fibrosis (PIPF), Obstructive sleep apnea, Polysomnography, Body mass index, Neck Circumference, Pulmonary Hypertension.

INTRODUCTION: Obstructive sleep apnea (OSA) has been shown to increase the risk for pulmonary hypertension, ischemic heart disease, stroke, congestive heart failure, and arrhythmias. ${ }^{1,2}$ Pulmonary artery pressure is increased during apneas, most likely due to pulmonary artery constriction induced by hypoxemia. The greatest increase occurs during rapid eye movement (REM) sleep, when hypoxemia is usually greatest. ${ }^{3}$ Cardiac arrhythmias are serious complications of obstructive sleep

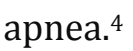

A pattern of repeated cycles of bradycardia during the apnea and followed by tachycardia with arousal that terminates the apnea is the most common. The mechanism for bradycardia appears to be a reflex increase in vagal tone caused by stimulation of carotid body receptors by hypoxia. Posttuberculosis can cause pulmonary hypertension due to extensive pulmonary fibrosis, which causes distortion of lung parenchyma. The basic underlying mechanism is hypoxia. ${ }^{5}$

Polysomnoraphy refer to the collective process of monitoring and recording physiological data during sleep. The variables monitored and recorded during sleep include Electroencephalography (EEG), Electro-oculogram (EOG), Electromyogram (EMG), Electro-cardiogram (ECG) Pulse-Oximeter. Obstructive sleep apnea and post-tuberculosis were associated with hypoxic complications. Hence, we conducted a clinical based study.

METHODOLOGY: Total 48 patients with post-infective pulmonary fibrosis with Obstructive sleep apnea were included in the study. The present study was conducted at Government medical college Akola from January 2014 to march 2015. X-Ray chest PA view, pulmonary function test, and echocardiography were done in selected patients and they were subjected by Polysomnoraphy and the results were noted. 


\section{ORIGINAL ARTICLE}

INCLUSION CRITERIA: Patients having lung conditions in which Forced Expiratory Volume in one second ( FEV1) less than $50 \%$ such as post-infective pulmonary fibrosis with symptoms suggestive of Obstructive sleep apnea.

\section{Exclusion Criteria:}

1. Chronic Obstructive Pulmonary Disease (COPD) with symptoms suggestive of Obstructive sleep apnea.

2. Bronchial asthma with symptoms suggestive of Obstructive sleep apnea.

3. Interstitial Lung Disease (ILD) with Obstructive sleep apnea symptoms.

An accurate patient history, including corroborative from bed partner and physical examination, paying particular attention to the head and neck circumference in the diagnosis of Obstructive sleep apnea was taken.

All patients were answered the questions about symptoms associated with sleep apnea, such as excessive daytime sleepiness, loud snoring, morning headache, irritability, difficulties with concentration and the Epworth sleepiness scale (ESS) was used to interpret the daytime sleepiness. ${ }^{6}$

Patients had symptoms of upper respiratory tract abnormality were referred to Ear, Nose and Throat specialist for examination of the upper airway.

Anthropometry height and weight of all patients were measured using standard equipment. Body Mass Index (BMI) is a patient's weight in kilograms divided by the square of height in meters.

Epworth sleepiness scales more than ten suggest clinically significant daytime sleepiness although the lower score does not exclude it. Sleep Study.7,8,9

An overnight sleep study was carried out using the E-series EEG/PSG comp medic's machine. The study was done in the sleep Laboratory.

\section{The Parameters were Monitored:}

- Electroencephalography (EEG): Using gold cup electrodes electric activity of the brain was monitored which were used for sleep staging. Electrodes were applied at the vertices and particular areas.

- Electro-oculogram (EEG): Gold up electrodes were applied around the eyes for recording ocular movements which help in differentiating Non-rapid eye movement (NREM) sleep from the Rapid eye movement (REM) stage of sleep

- Electromyogram (EMG): Electrodes were applied over the chin muscles to record the muscle movements.

- $\quad$ Electrocardiogram (ECG): Electrodes use to record heart rate.

- Pulse Oximeter: Pulse-Oximeter was used to monitor the trends of oxygen saturation during overnight sleep study.

- Thermistor based airflow sensor used to detect respiratory airflow.

- Thoracic and abdominal movements during sleep study were recorded using abdominal and thoracic bands.

- Body position sensor: This helps to detect the effects of change of position on apnea episodes.

- $\quad$ PLM sensors: These monitor the leg movements during sleep and any arousals associated 


\section{ORIGINAL ARTICLE}

with increase leg movements. It plays an important role in diagnosing restless leg syndrome (RLS).

- $\quad$ Nocturnal desaturation was defined as oxygen saturation less than $90 \%$ by pulse Oximeter for more than $10 \%$ of the total sleep time.

RESULTS AND ANALYSIS: Apnea-hypopnea index (AHI) less than 5 was considered normal. An apnea-hypopnea index of 5-14 was mild, 15-29 was moderate and equal or more than 30 events per hours characterized severe sleep apnea.

Correlation of demographic parameters with the severity of obstructive sleep apnea.

Out of 48 patients $7(14 \%), 3(6 \%), 2(4 \%), 1(2 \%)$ patients in the age group of $20-40$ had apnea-hypopnea index less than 5,5-14, 15-29 and equal or more than 30 respectively, similarly $16(32 \%), 5(10 \%), 4(8 \%), 2(4 \%)$ patients in the age group of 40-60 had apnea-hypopnea index less than 5, 5-14, 15-29 and equal or more than 30 respectively. In the age group of $60-803(6 \%), 2(4 \%)$, $2(4 \%), 1(2 \%)$ patients had apnea-hypopnea index less than 5, 5-14,15-29 and equal or more than 30 respectively as shown in figure 1 and statistically significant with $P$ value 0.001 .

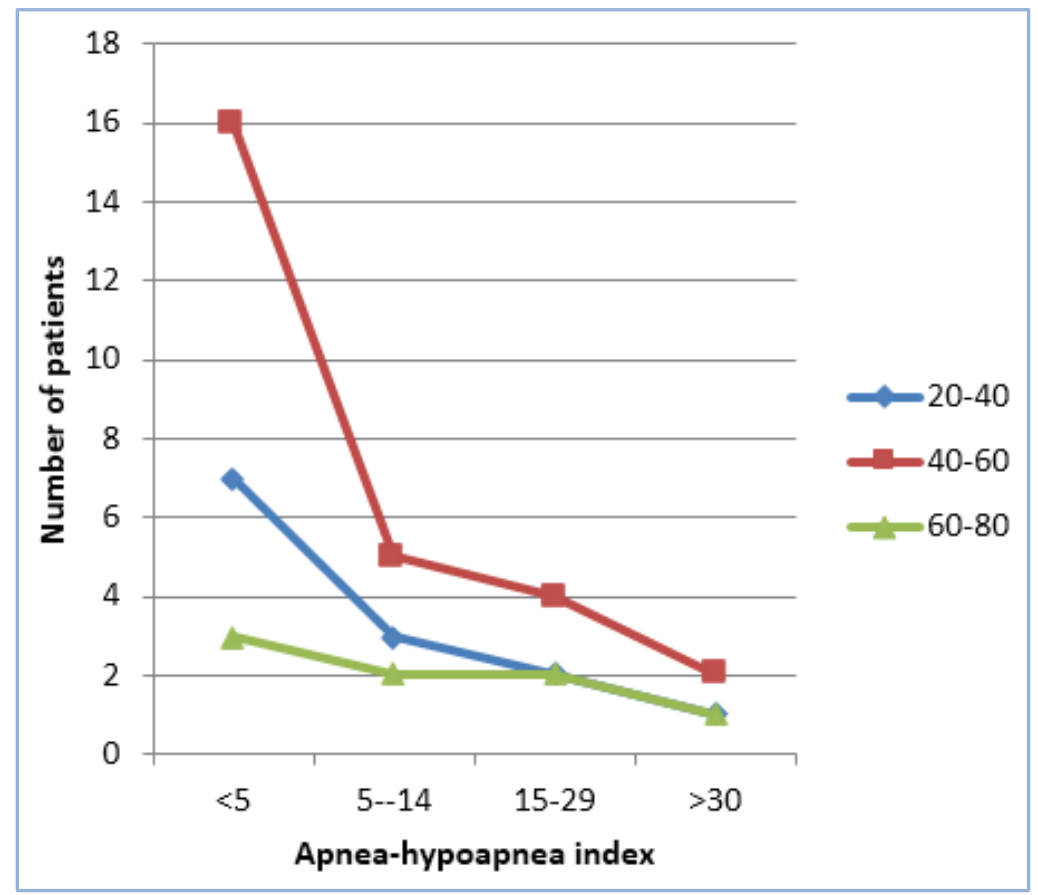

Fig. 1: Age wise Distribution

Out of 48 patients, 38(76\%) were males and 10(20\%) were female patients. Out of 38 males, $20(40 \%)$ had apnea-hypopnea index less than 5, 8(16\%) had apnea-hypopnea index 5-14, 7 (14\%) had AHI 15-29 and $3(6 \%)$ had AHI equal or More than 30.0ut of 10 female patients $7(14 \%)$ had apnea-hypopnea index less than 5, 2(4\%) had AHI between 5-14,1(2\%) patients had AHI 15-29.It's P Value 0.30 not significant. 


\section{ORIGINAL ARTICLE}

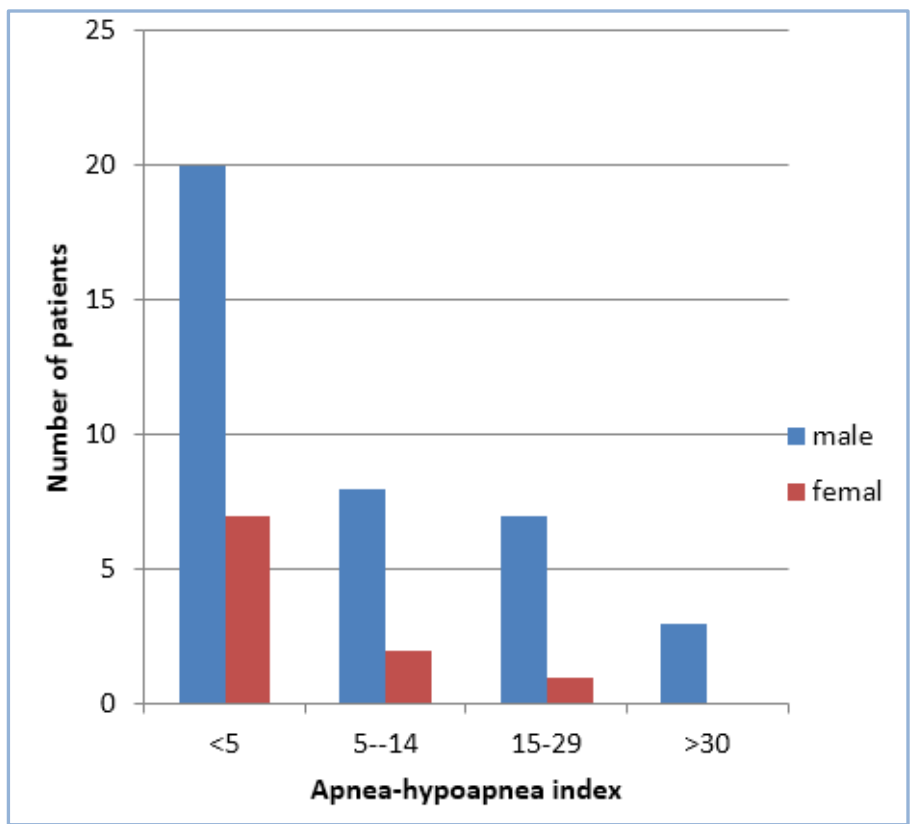

Fig. 2: Sex wise Distribution

Correlation of respiratory disease with Obstructive sleep apnea to apnea-hypopnea index.

Out of 48 patients, $27(54 \%)$ patients with post-infective pulmonary fibrosis had apneahypopnea index less than five, 10(20\%) patients had apnea-hypopnea index 5-14, 8(16\%) had apneahypopnea index 15-29 and 3(6\%) had AHI equal or more than 30.

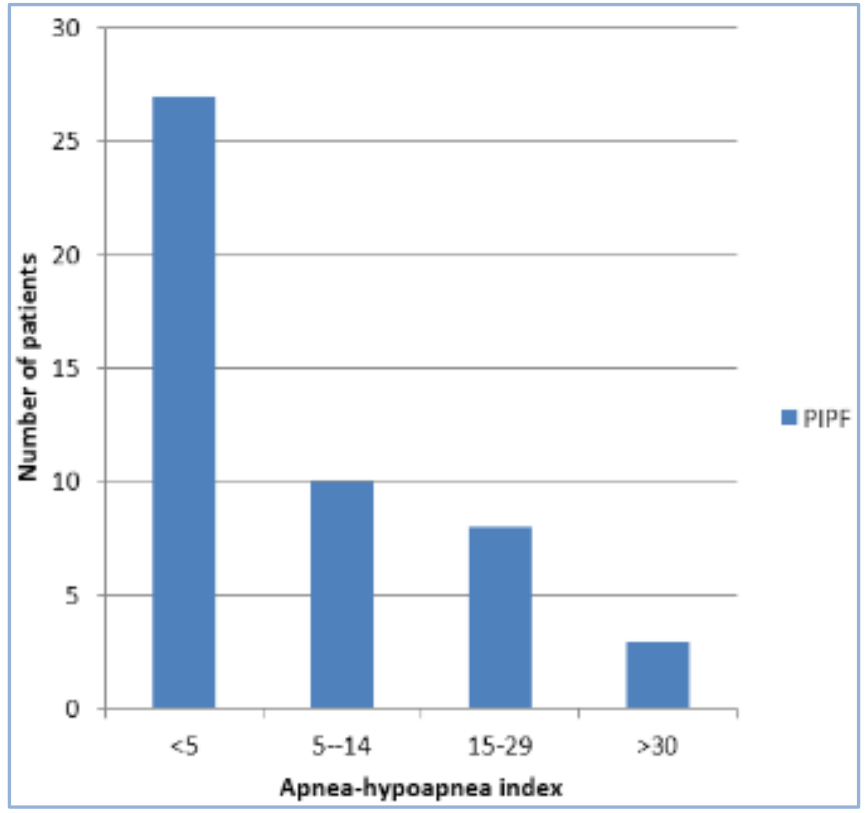

Fig. 3: Distribution of Post-infective pulmonary fibrosis with obstructive sleep apnea pattern 


\section{ORIGINAL ARTICLE}

Correlation of morphological parameters to obstructive sleep apnea.

Certain body morphological characters like body mass index and neck circumference were measured and the observations were correlated with the severity of obstructive sleep apnea. As body mass index increases the severity of obstructive sleep apnea increases.

There were 16 total number of patients with body mass index of less than 25 , out of them $14(28 \%), 1(2 \%), 1(2 \%)$, had apnea-hypopnea index less than 5, 5-14 and 15-29 respectively.19 patients had body mass index in the range of 25-30, out of them $11(22 \%), 4(8 \%), 3(6 \%), 1(2 \%)$ patients had apnea-hypopnea index less than 5, 5-14, 15-29 and equal or more than 30 respectively, there were $8(16 \%)$ patients with body mass index in the range of $30-40$ out of them $1(2 \%), 3(6 \%)$, $3(6 \%)$ and $1(2 \%)$ had apnea-hypopnea index less than 5,5-14,15-29 and equal or more than 30 respectively, with body mass index more than 40,there were $1(2 \%), 2(4 \%), 1(2 \%), 1(2 \%)$ patients had apnea-hypopnea index less than 5,5-14,15-29 and equal or more than 30 respectively. It was statistically significant as a P value less than 0.001 .

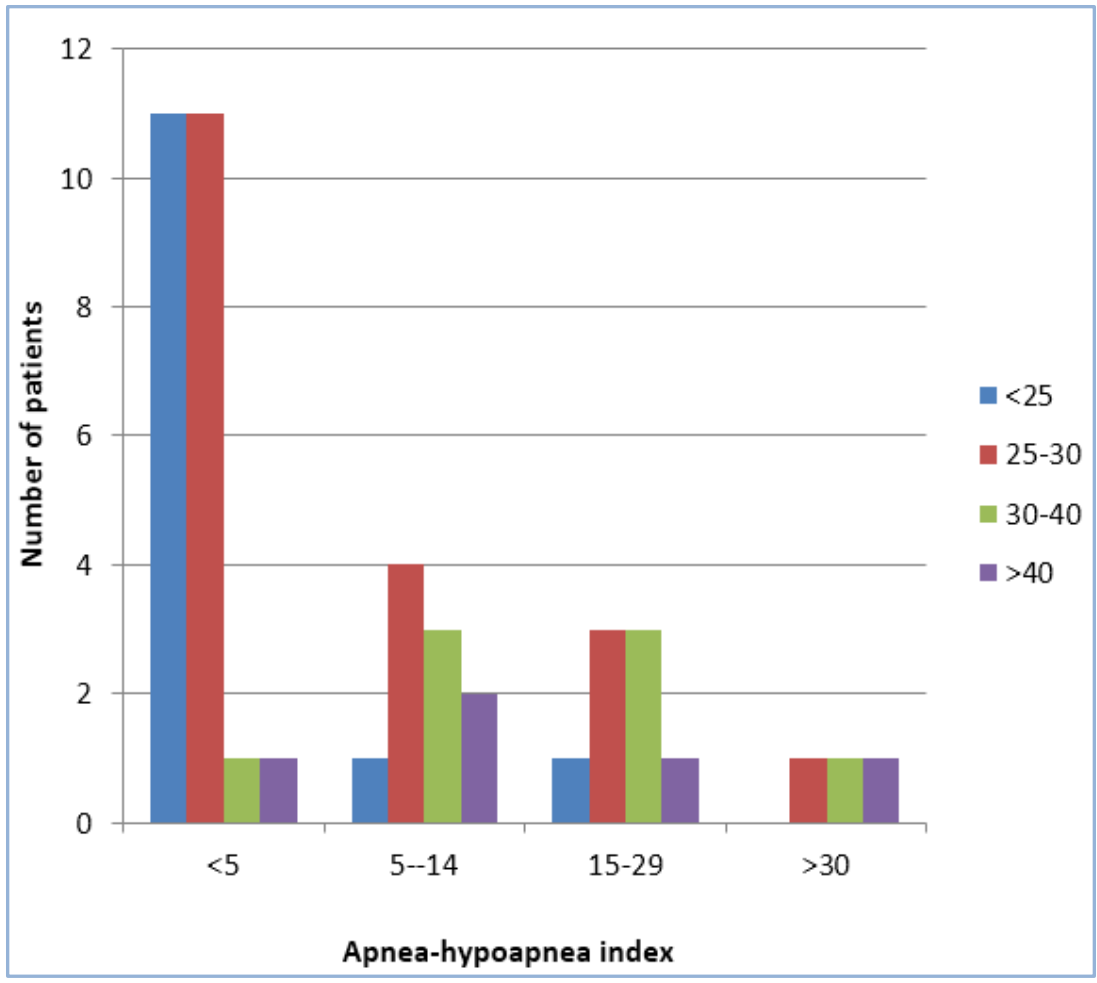

Fig. 4: Correlation of body mass index with apnea-hypopnea index.

As neck circumference increases the severity of OSA increases. Total $16(32 \%)$ patients were present with neck circumference in the range of $10-15$,out of them $11(22 \%), 3(6 \%), 1(2 \%), 1(2 \%)$ had apnea-hypopnea index less than 5, 5-14, 15-29 and equal or more than 30 respectively. 27(54\%) patients were present in the range of $15-20$, out of them $15(30 \%), 5(10 \%), 5(10 \%)$ and $2(4 \%)$ had apnea-hypopnea index less than 5, 5-14, 15-29 and equal or more than 30 respectively. With a neck circumference more than 20 there were 1(2\%), 2(4\%), 1(2\%), 1(2\%) had apnea-hypopnea index less than $5,5-14,15-29$ and equal or more than 30 respectively.

It was not statistically significant as $P$ value 0.70 . 


\section{ORIGINAL ARTICLE}

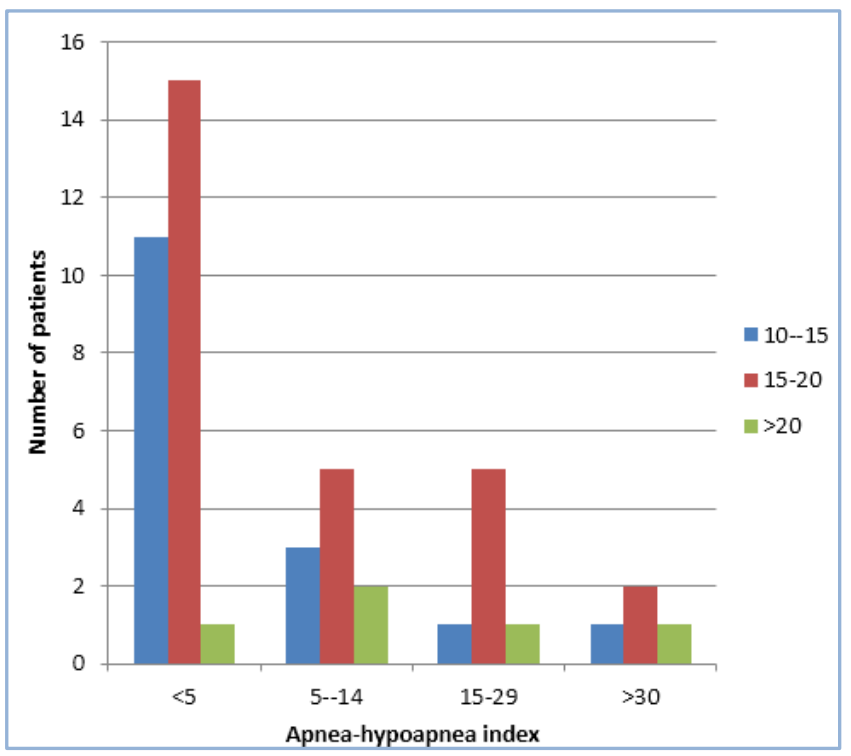

Fig. 5: Correlation of neck circumference with apnea-hypopnea index.

Correlation of the Post-infective pulmonary fibrosis with obstructive Sleep apnea to desaturation.

Patients of Post-infective pulmonary fibrosis with Obstructive sleep apnea were shows desaturation. Out of 48 patients, $40(80 \%)$ patients had saturation between $91-94 \%, 6(12 \%)$ had desaturation between $75-90 \%$, and 2 (4\%) patients had desaturation less than $75 \%$.

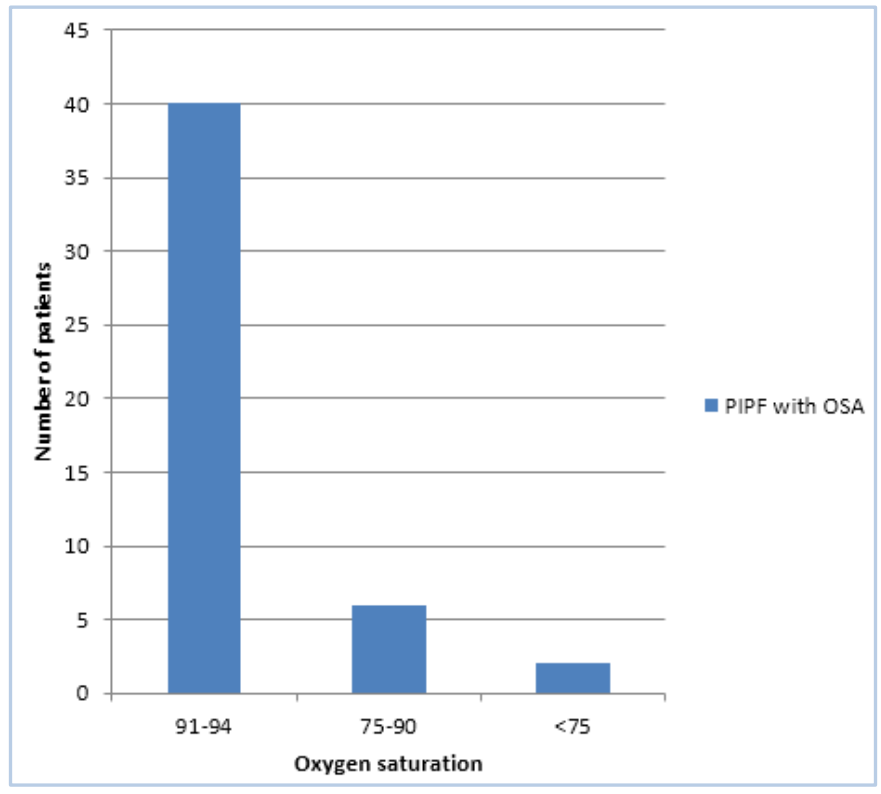

Fig. 6: Correlation of Post-infective pulmonary fibrosis with Obstructive sleep apnea to Desaturation 


\section{ORIGINAL ARTICLE}

Correlation of the Post-infective pulmonary fibrosis with Obstructive sleep apnea to pulmonary hypertension.

Out of 48 patients,13(26\%) patients had pulmonary hypertension, out of them $4(8 \%)$ patients had apnea-hypopnea index less than 5, and 3(6\%), 4(8\%), 2(4\%) patients had apneahypopnea index between 5-14,15-29,equal or more than 30 respectively, Not statistically significant as $P$ value 0.50 .

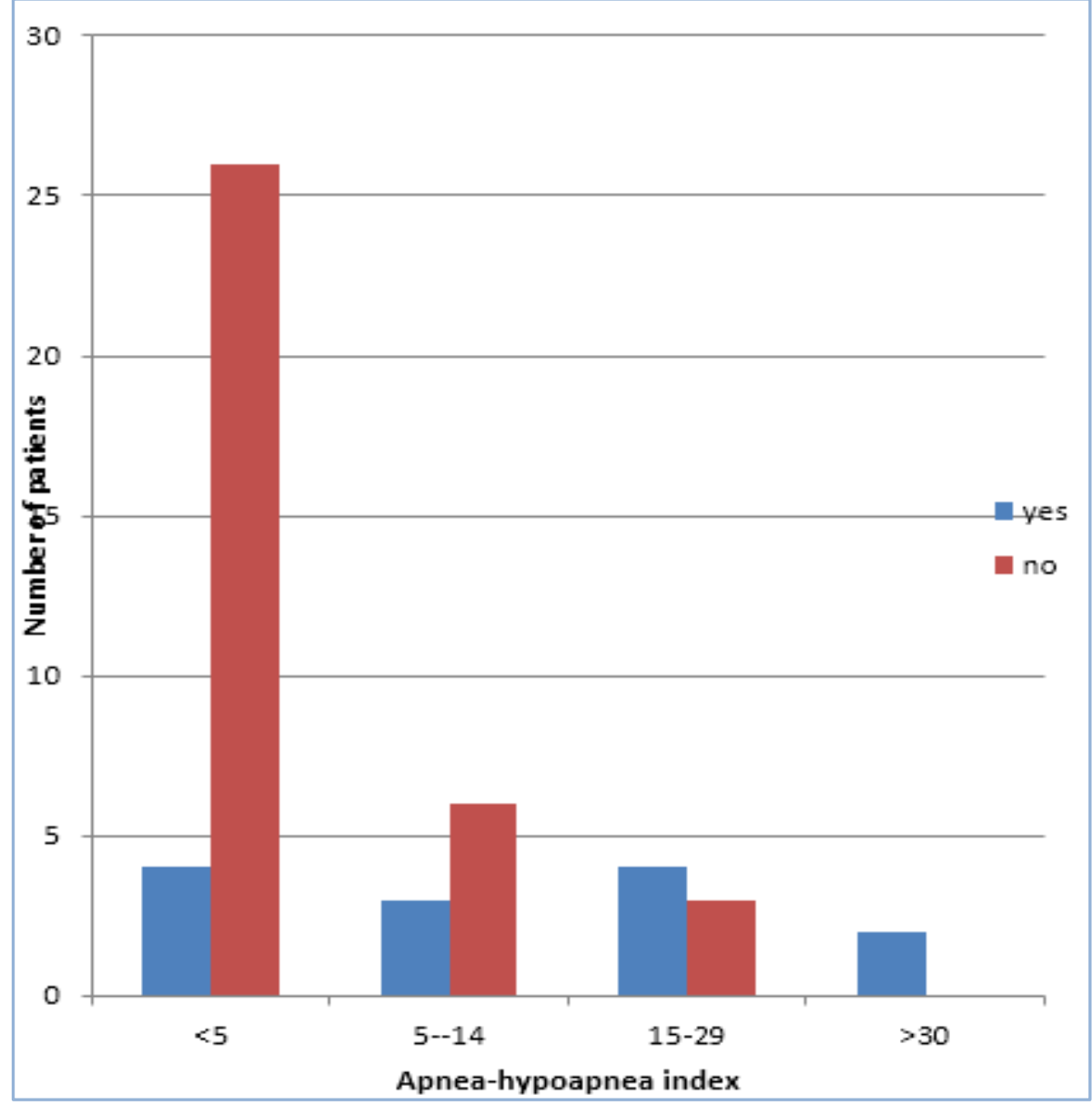

Fig. 7: Distribution of pulmonary hypertension in patients with Post-infective pulmonary Fibrosis with Obstructive sleep apnea.

DISCUSSION: In the present study total number of patients were 60 among those 12 patients were not followed hence 48 patients were selected in this study, they were evaluated for sleep disordered breathing, 48 patients were Post-infective pulmonary fibrosis with Obstructive sleep apnea. This study provided data on occurrence of obstructive sleep apnea in respiratory diseases and its association with a variety of potential causes such as body mass index, desaturation, pulmonary hypertension.

Conventional measures apnea-hypopnea index was used to define the presence of obstructive sleep apnea. In our study apnea-hypopnea index more than 5 with at least two symptoms were considered as obstructive sleep apnea-hypopnea syndrome. Various investigators have taken a 


\section{ORIGINAL ARTICLE}

different level of apnea-hypopnea index cut off point of 5, 14, 29 events per hours to indicate mild, moderate and severe levels of Obstructive sleep apnea. Out of 48 patients included in this, study $38(76 \%)$ patients were males and $10(20 \%)$ females, of 38 males $18(36 \%)$ patients had Obstructive sleep apnea while 3(6\%) patients out of 10 females had Obstructive sleep apnea.

There was no sexual predilection conforming incidence of Obstructive sleep apnea in the patients in their fifth to seventh decade of life, which corroborates to the study of Ancolis Israel et al \& Bixter et al.

The study found that 19 patients out of 48 patients were overweight,16(32\%) patients had a body mass index less than $25,19(38 \%)$ patients had a body mass index between $25-30,8(16 \%)$ patients between 30-40 and $5(10 \%)$ had body mass index more than 40 .

Young et al found that an increase in body mass index more than one standard deviation increase in the chances of obstructive sleep apnea by four fold. ${ }^{10}$ Guill eminault et al their study found that three fourth of patients with Obstructive sleep apnea were obese. In our study, we found that as body mass index increase the severity of obstructive sleep apnea also increases.

Stradling et al in their study found a clear relationship between excess body weight usually expressed as body mass index and number of sleep related respiratory disturbances, which corroborates with our study.

Anthropometric evaluation in the form of neck circumference has been found to be good predictors of Obstructive sleep apnea. Obstructive sleep apnea has been identified as an independent risk factor for hypertension with a linear relationship exist between pulmonary hypertension and Obstructive sleep apnea.

In our study found that there is an association between pulmonary hypertension and Obstructive sleep apnea with post-infective pulmonary fibrosis.11,12 The relationship between pulmonary hypertension and Obstructive sleep apnea is partly due to confounding by the common risk factors (obesity and age). ${ }^{13}$

Eight (16\%) post-infective pulmonary fibrosis patients with Obstructive sleep apnea had hypoxemic episodes during wakefulness become more hypoxic during sleep. ${ }^{14,15,16,17,18}$ More severe episodes of nocturnal desaturation generally occur during rapid eye movement sleep. ${ }^{19,20}$ The consequence of sleep related hypoxemia includes peak pulmonary hypertension due to hypoxemic pulmonary vasoconstriction. ${ }^{21}$

SUMMARY AND CONCLUSION: In summary, this study suggests the prevalence of sleep apnea in Post-infective pulmonary fibrosis and association of pulmonary hypertension, desaturation in patients with Post-infective pulmonary fibrosis with Obstructive sleep apnea:

1. Sleep disordered breathing is more prevalent in Post-infective pulmonary fibrosis with obstructive sleep apnea.

2. The occurrence of pulmonary hypertension is more in patients of Post-infective pulmonary fibrosis with Obstructive sleep apnea.

3. Post-infective pulmonary fibrosis with Obstructive sleep apnea shows more desaturation.

4. Higher body mass index is associated with higher apnea -hypopnea index.

\section{Abbreviations:}

OSA Obstructive sleep apnea.

OSAHS Obstructive sleep apnea-hypopnea syndrome. 


\section{ORIGINAL ARTICLE}

AHI apnea -hypopnea index.

PIPF Post-infective pulmonary fibrosis.

BMI Body mass index.

NC Neck Circumference.

REM sleep, Rapid eye movement sleep.

NREM sleep Non rapid eye movement sleep.

\section{REFERENCES:}

1. Carpio C, Alvarez-Sala R, Garcia-Rio F. Epidemiological and pathogenic relationship between sleep apnea and ischemic heart disease. Pulm Med 2013; 2013:405827.

2. Ali Lk, Avidan AY. Sleep disordered breathing and stroke. Rev Neurol Dis 2008; 5(4):191-8.

3. Levy P, Pepin JL, Arnaud C, et al. Intermittent hypoxia and sleep disordered breathing: current concepts and perspectives. Eur Respir J 2008; 32(4):1082-95.

4. Shamsuzzaman As, Gersh BJ, Somers VK. Obstructive sleep apnea: implications for cardiac and vascular disease. JAMA 2003; 290(14):1906-14.

5. Farber HW, Loscalzo J. Pulmonary arterial hypertension Eng J Med.2004; 351:1655-65.

6. Johns MW. A new method for measuring daytime sleepiness: The Epworth Sleepiness Scale. Sleep 1991; 14(6):540-5.

7. American College of Occupational Environmental Medicine Presidential Task force on fatigue Risk Management (Feb 2012) "Fatigue risk Management in the workplace". Journal of Occupational and Environmental Medicine 54 (2): 231-58.

8. Rechtschanffen, A, A \& Kales, A. (EDS.) (1968). A manual standardized Terminology, techniques, and scoring system for sleep stages of human Subjects. Washington, D.C.: Public health services, U S Government Printing service.

9. Berry, Richard et al, (2012). A The AASM Manual for the scoring of Sleep and Associated Events; Rules Terminology and Technical Specifications, Version 2.0.Darien, IL; American Academy of Sleep Medicine.

10. Young T, Peppard P, Palta M, Hla KM, Finn L, Morgan B, Skatrud J. Population-based study of sleep-disordered breathing as a risk factor for Hypertension. Arch Intern Med 1997; 157:17461752. (Pub Med)

11. Motley HL, Coumand A, Werko L, Himmelstein A, Dresdale D, The Influence of short periods of inducing acute anoxia upon pulmonary artery Pressures in man. Am J Physiol 1947; 150:315320 ?

12. Sanner BM, Doberauer C, Konermann M, Sturm A, Zidek W. pulmonary Hypertension in patients with obstructive sleep apnea syndrome. Arch Intern Med.1997; 157:2483-7.

13. Mc Quillan BM, Picard MH, Leavitt M, Weyman AE. Clinical correlates and Reference intervals for pulmonary artery systolic pressure among Echocardiographically normal subjects. Circulation.2001 Dec4; 104(23):2797-802.Pub Med.

14. Bradley TD, Rutherford R, Grossman RF, et al. Role of daytime hypoxia in The pathogenesis of right heart failure in obstructive sleep apnea Syndrome. Am Rev Respir Dis.1985; 161:835-9.

15. Sajkov D, Cowie RJ, Thornton AT, Espinoza HA, McEvoy RD. Pulmonary hypertension and hypoxemia in obstructive sleep apnea Syndrome. Am J Respir Crit Care Med.1994; 149:416-22.

16. Bady E, Achkar, Pascal S, Orvoen-Frija E, Laaban Jp. Pulmonary arterial Hypertension in patients with sleep apnea syndrome.Thorax.20000:55:934-9 


\section{ORIGINAL ARTICLE}

17. KriegerJ, Sforza E, Apprill M, Lampert E, Weitzenblum E, Ratomaharo J. pulmonary hypertension, hypoxemia, and hypercapnia in obstructive Sleep apnea patients. Chest. 1989; 96:729-37.

18. Chaouat A. Weitzemblum E, KriegerJ, Ifoundza T, Oswald M., Kessler R. Association of chronic obstructive pulmonary disease and sleep apnea Syndrome. Am J Respir Crit care Med.1995; 151:82-6.

19. Zhonghua Nei za Zhi 2007 Jun; 46 (6):458-61.

20. Sleep 2012 Jan 1; 35(1):89-96.doi:105665/sleep.1592 Ling IT, James AL Hillman Dr PMID: 22215922 (Pub MED-indexed for MEDLINE) PMCID.

21. Voelkel NF. Mechanism of hypoxic pulmonary vasoconstriction. AM, Rev Respir Dis 1986; 133:1186-1195 intervals for pulmonary artery systolic Pressure among echocardiographicall normal subjects. Circulation 2001; 104:2797-2802.

\section{AUTHORS:}

1. Bhise Dhondiba Haribhau

\section{PARTICULARS OF CONTRIBUTORS:}

1. Assistant Professor, Department of Pulmonary Medicine, Government Medical College and Hospital, Akola.

FINANCIAL OR OTHER

COMPETING INTERESTS: None
NAME ADDRESS EMAIL ID OF THE CORRESPONDING AUTHOR:

Dr. Bhise Dhondiba Haribhau, Room No 27 (A), Ground Floor, Government Medical officer Boys Hostel \& Main Hospital, Akola.

E-mail: makayeegate@gmail.com

Date of Submission: 03/07/2015. Date of Peer Review: 04/07/2015. Date of Acceptance: 13/08/2015. Date of Publishing: 18/08/2015. 